\title{
Sleep deprivation and Modafinil affect cortical sources of resting state electroencephalographic rhythms in healthy young adults
}

\author{
Claudio Del Percio ${ }^{\mathrm{a}}$, Philippe Derambure ${ }^{\mathrm{b}}$, Giuseppe Noce ${ }^{\mathrm{c}}$, Roberta Lizio ${ }^{\mathrm{c}}$, David Bartrés-Faz ${ }^{\mathrm{d}}$, \\ Olivier Blin ${ }^{\mathrm{e}}$, Pierre Payoux ${ }^{\mathrm{f}}$, Dominique Deplanque ${ }^{\mathrm{g}}$, Déborah Méligne ${ }^{\mathrm{h}}$, Nicolas Chauveau ${ }^{\mathrm{h}}$, \\ Jean Louis Bourriez ${ }^{\mathrm{b}}$, Catherine Casse-Perrot ${ }^{\mathrm{e}}$, Laura Lanteaume ${ }^{\mathrm{i}}$, Claire Thalamas ${ }^{\mathrm{j}}$, \\ Juergen Dukart ${ }^{\mathrm{k}}$, Raffaele Ferri ${ }^{1}$, Maria Teresa Pascarelli ${ }^{1}$, Jill C. Richardson ${ }^{\mathrm{m}}$, \\ Regis Bordet $^{\mathrm{b}}$, Claudio Babiloni ${ }^{\mathrm{a}, \mathrm{n}, *}$, on behalf of the PharmaCog Consortium \\ a Department of Physiology and Pharmacology "Erspamer", Sapienza University of Rome, Rome, Italy \\ ${ }^{\mathrm{b}}$ Univ Lille, Inserm, CHU Lille, UMR_S 1171 - Degenerative and Vascular Cognitive Disorders, F59000 Lille, France \\ ${ }^{\mathrm{c}}$ IRCCS SDN, Napoli, Italy

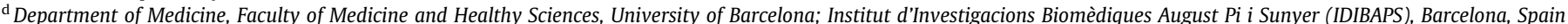 \\ e Aix Marseille University, UMR-CNRS 7289, Service de Pharmacologie Clinique, AP-HM, Marseille, France \\ ${ }^{\mathrm{f}}$ INSERM, Imagerie Cérébrale et Handicaps Neurologiques, Toulouse, France \\ ${ }^{\mathrm{g}}$ Univ Lille, Inserm, CHU Lille, CIC1403 E UMR S 1171 - Degenerative and Vascular Cognitive Disorders, F59000 Lille, France \\ ${ }^{\mathrm{h}}$ INSERM UMR 825 Brain Imaging and Neurological Dysfunctions, Toulouse, France \\ ${ }^{\mathrm{i}}$ Service de Neurologie et Neuropsychologie, APHM Hôpital Timone Adultes, Marseille, France \\ ${ }^{j}$ Department of Medical Pharmacology, INSERM CIC 1436, Toulouse University Medical Center, Toulouse, France \\ ${ }^{\mathrm{k}}$ F. Hoffmann-La Roche, Pharma Research Early Development, Roche Innovation Centre Basel, Basel, Switzerland \\ ${ }^{1}$ Oasi Research Institute - IRCCS, Troina, Italy \\ ${ }^{\mathrm{m}}$ Neurosciences Therapeutic Area, GlaxoSmithKline RED, Stevenage, UK \\ ${ }^{\mathrm{n}}$ Hospital San Raffaele Cassino, Cassino, FR, Italy
}

\section{A R T I C L E I N F O}

\section{Article history:}

Accepted 3 June 2019

Available online 1 July 2019

Keywords:

Resting state electroencephalographic

(rsEEG) rhythms

Sleep deprivation

Modafinil

Alzheimer's disease (AD)

Exact low-resolution brain electromagnetic

source tomography (eLORETA)

\section{H I G H L I G H T S}

- Sleep deprivation (SD) affects resting EEG sources in healthy subjects.

- Modafinil partially recovers those SD effects.

- Those EEG sources are related to brain arousal and vigilance.

\begin{abstract}
A B S T R A C T
Objective: It has been reported that sleep deprivation affects the neurophysiological mechanisms underpinning the vigilance. Here, we tested the following hypotheses in the PharmaCog project (www.pharmacog. org): (i) sleep deprivation may alter posterior cortical delta and alpha sources of resting state eyes-closed electroencephalographic (rsEEG) rhythms in healthy young adults; (ii) after the sleep deprivation, a vigilance enhancer may recover those rsEEG source markers.

Methods: rsEEG data were recorded in 36 healthy young adults before (Pre-sleep deprivation) and after (Postsleep deprivation) one night of sleep deprivation. In the Post-sleep deprivation, these data were collected after a single dose of PLACEBO or MODAFINIL. rSEEG cortical sources were estimated by eLORETA freeware. Results: In the PLACEBO condition, the sleep deprivation induced an increase and a decrease in posterior delta $(2-4 \mathrm{~Hz})$ and alpha $(8-13 \mathrm{~Hz})$ source activities, respectively. In the MODAFINIL condition, the vigilance enhancer partially recovered those source activities.

Conclusions: The present results suggest that posterior delta and alpha source activities may be both related to the regulation of human brain arousal and vigilance in quiet wakefulness.
\end{abstract}

\footnotetext{
* Corresponding author at: Department of Physiology and Pharmacology “V. Erspamer”, University of Rome "La Sapienza”, P. le A. Moro 5, 00185 Rome, Italy.

E-mail address: claudio.babiloni@uniroma1.it (C. Babiloni).
} 
Significance: Future research in healthy young adults may use this methodology to preselect new symptomatic drug candidates designed to normalize brain arousal and vigilance in seniors with dementia. (C) 2019 International Federation of Clinical Neurophysiology. Published by Elsevier B.V. All rights

\section{Introduction}

Alzheimer's disease $(\mathrm{AD})$ is a chronic neurodegenerative disease characterized by cognitive (e.g. typically memory loss), functioning, and behavioral abnormalities. The typical amnesic manifestation of the $A D$ is related to an impairment of the cholinergic basal forebrain, thalamocortical system, associative parietaltemporal areas, and the circuits linking the hippocampus, entorhinal cortex, and amygdala (Daulatzai, 2010). To date, there are no disease-modifying drugs that can prevent, care or even slow down the $\mathrm{AD}$ pathological processes. Instead, there are two therapeutic classes licensed for the symptomatic treatment of the cognitive deficits in $\mathrm{AD}$, namely the acetylcholinesterase inhibitors and a NMDA receptor glutamatergic antagonist. Unfortunately, these drugs have only modest effects on AD symptoms, and more research is needed to advancing the treatment of the disease (Babiloni et al., 2013a).

How to improve the early stages of the AD drug discovery pathway? This objective may be achieved by procedures showing that a given compound induces a beneficial recovery in healthy young volunteers from an alteration of brain activity and cognitive processes, similar to those observed in the disease, artificially due to a challenging procedure (Widlöcher, 1996; Babiloni et al., 2014a). In principle, this kind of challenge models may overcome the inherent difficulty of detecting significant improvements in brain activity and cognitive performance in normal healthy subjects. Examples of this kind of challenges have previously been limited to pharmacological interventions such as the administration of antagonists of the cholinergic or glutamatergic neurotransmission such as scopolamine or ketamine (Ebert et al., 2001; Snaedal et al., 2010; Horacek et al., 2010).

Sleep deprivation shows features of interest to be considered as a potential transient and reversible challenge model for the symptomatic drug discovery pathway. Indeed, a bulk of previous studies revealed that one night or more of sleep deprivation does induce an alteration of vigilance (Cassé-Perrot et al., 2016). This alteration would deteriorate cognitive processes such as executive functions and attention, working and episodic memory, visuospatial abilities, and language as a function of the sleep deprivation duration, task difficulty, the procedures for the measurement of cognition, gender, and subject's age (Killgore et al., 2008; Cassé-Perrot et al., 2016). A pharmacological intervention with caffeine (i.e. blocking of adenosine receptors and inhibition of phosphodiesterase), dextroamphetamine (i.e. norepinephrine and dopamine agonist), and Modafinil (i.e. reuptake inhibitor dopamine agonist) restored the alertness in the Post-sleep deprivation period in a complex manner with reference to the kind of the cognitive demands (Killgore et al., 2009) and gender. The Modafinil had clear effects on both women and men (Killgore et al., 2008).

It is well known that vigilance in the sleep-wake axis affects psychomotor performance and is influenced by the interaction of a sleep/wake dependent homeostatic process and a circadian process of general arousal and body temperature ( Achermann, 2004; Van Dongen and Dinges, 2005). The sleep homeostatic process induces a pressure for sleeping during the wakefulness, which is dissipated during the sleep. The circadian rhythms yield a waning and waxing of pressure for the wakefulness over the day. During total sleep deprivation, these two processes cause psychomotor performance to deteriorate over time.

The effects of the sleep deprivation on the neurophysiological mechanisms underpinning the vigilance were mostly unveiled by studies using the recording of resting state electroencephalographic (rsEEG) rhythms. It has been reported that the sleep deprivation for 38-40 $\mathrm{h}$ altered the amplitude (power) of the scalp rsEEG rhythms in the relaxed wakefulness at some specific frequency bands such as delta $(<4 \mathrm{~Hz})$, theta $(4-8 \mathrm{~Hz})$, alpha $(8-$ $12 \mathrm{~Hz}$ ), and beta $(12.25-25.0 \mathrm{~Hz}$ ) rhythms (Cajochen et al., 1995; Corsi-Cabrera et al., 1996, 2003; Aeschbach et al., 1997; Dumont et al., 1999; Mander et al., 2010). Delta and theta rhythms were characterized by a similar Post-sleep deprivation time course, reflecting both a circadian modulation and the duration of the time awake (Aeschbach et al., 1997; Dumont et al., 1999; Cajochen et al., 2001; Corsi-Cabrera, 2003). Less clear was that correlation for the alpha rhythms, due to a possible nonlinear interaction between the homeostatic sleep process and the circadian rhythms (Aeschbach et al., 1997; Dumont et al., 1999). The power of the beta rhythms exhibited a wake-dependent increase (Aeschbach et al., 1997; Dumont et al., 1999). Noteworthy, the sleep deprivation increased the power of the theta rhythms and decreased that of the alpha rhythms (Dumont et al., 1999; Corsi-Cabrera, 2003).

The clinical neurophysiological interest of the sleep deprivation challenge relies on the fact that its effect on rsEEG rhythms is reminiscent of that of AD (Giaquinto and Nolfe, 1986; Breslau et al., 1989; Briel et al., 1999). Previous studies have shown that the power (i.e. spectral power density) of eyes-closed rsEEG rhythms was abnormal in patients with $\mathrm{AD}$ and amnesic mild cognitive impairment (MCI) as a prodromal stage of the disease (Lehmann et al., 2007; Bonanni et al., 2008; Ommundsen et al., 2011). Compared with healthy subjects, $\mathrm{AD}$ and $\mathrm{MCI}$ patients were characterized by higher power of the widespread delta rhythms $(0-4 \mathrm{~Hz})$ while a lower power was found in the posterior alpha rhythms (8-12 Hz; Huang et al., 2000; Dierks et al., 1993; Jeong, 2004; Moretti et al., 2004). Even whether those rsEEG rhythms may not directly reflect the specific pathophysiological markers of $A D$ (Dubois et al., 2014), they are promising topographical markers indexing the thalamocortical functional reserve underpinning the regulation of brain arousal in quiet vigilance.

An important methodological limitation is that rsEEG scalp topography is influenced by the blurring effects of the reference electrode and head volume conduction (Nunez, 1987). To mitigate this limitation, a promising approach stems upon an estimation of cortical sources of eyes-closed rsEEG rhythms by the lowresolution brain electromagnetic tomography (LORETA). LORETA uses a brain source space coregistered to Talairach brain atlas that is typically adopted in neuroimaging studies in humans (Pascual-Marqui et al., 1994).

LORETA methodology has been extensively used in the EEG module of the European FP7-IMI (Innovative Medicine Initiative) project with the short title "PharmaCog" (2010-2015; www.pharmacog.org). In the PharmaCog EEG studies, we have shown that in $\mathrm{AD}$ patients at the stages of mild cognitive impairment (ADMCI) and dementia (ADD), posterior cortical sources of delta $(2-4 \mathrm{~Hz})$ and alpha $(8-13 \mathrm{~Hz})$ rhythms recorded in quiet wakefulness were abnormal. More specifically, compared with normal elderly 
subjects, ADMCI and ADD patients were characterized by less reduction (reactivity) in cortical alpha sources from eyes-closed to -open in the resting state condition, namely an experimental paradigm modulating subject's vigilance (Babiloni et al., 2010). Furthermore, posterior delta and alpha cortical source activities in the eyes-closed condition were more abnormal in ADD than $\mathrm{ADMCI}$ patients and were linearly correlated with the atrophy of normalized cortical gray matter and measurements of global cognitive status (Babiloni et al., 2013). Moreover, posterior cortical sources of delta and alpha rhythms in the eyes-closed condition were more abnormal in $\mathrm{ADMCI}$ than $\mathrm{MCI}$ patients not suffering from $\mathrm{AD}$, thus confirming the strict relationship of those EEG source alterations and disease neuropathology (Galluzzi et al., 2016; Jovicich et al., 2019).

Unfortunately, those previous PharmaCog findings did not clarify whether abnormal posterior cortical delta sources in $\mathrm{AD}$ patients may be related to changes in the level of vigilance in quiet wakefulness, as a possible reflection of the effects of disease neuropathologies on neurophysiological mechanisms regulating brain arousal. Traditionally, cortical delta rhythms are considered as an epiphenomenon in healthy subjects resting in quiet wakefulness, so the mentioned abnormalities in posterior cortical delta sources may merely reflect brain neuropathology in AD patients without implications on the regulation of the vigilance. This issue is clearly relevant for the interpretation of the effects of an intervention on posterior cortical delta sources in testing new symptomatic drugs improving vigilance in $\mathrm{AD}$ patients.

Keeping in mind the above open issue, the present study tested the following two hypotheses: (i) one night of sleep deprivation may alter cortical sources of resting state eyes-closed electroencephalographic (rsEEG) rhythms in healthy young adults; (ii) after the sleep deprivation (Post-sleep deprivation), a single dose of a drug enhancing vigilance (Modafinil) may recover those rsEEG source markers. These hypotheses globally evaluated if rsEEG source activities at delta $(2-4 \mathrm{~Hz})$ and alpha $(8-13 \mathrm{~Hz})$ frequencies were both related to the regulation of human brain arousal and vigilance in quiet wakefulness.

\section{Materials and Methods}

\subsection{Subjects}

The sample size calculation was based on previous evidence showing the effect of sleep deprivation on cognitive tasks in humans (Groeger et al., 2008; Lo et al., 2012). The expected effect size was set to 0.70 . The number of subjects estimated was of 27 , considering a one-sided alpha level of 0.05 , a power of $80 \%$, and the mentioned cross-over design. Estimating a subjects' withdrawn rate of about $25 \%$, the sample size of 36 right-handed (male) healthy young subjects was determined. In this line, 36 healthy volunteers, right handed were enrolled. These subjects were recruited by the following qualified clinical recording units of the PharmaCog project: Universities of Lille 2, Toulouse, and Marseille (France). At this early stage of the research, we selected only male subjects to obtain results comparable with those of previous reference studies on the effects of sleep deprivation and Modafinil on rsEEG rhythms (i.e. Chapotot et al., 2003, Bodenmann et al., 2009; James et al., 2011). Furthermore, we did not enroll female subjects to avoid (i) interactions between sleep deprivation, Modafinil, and menstrual cycle and (ii) possible effects of the sleep deprivation and the pharmacological manipulation on embryos in case of unaware conception in the long period of the experiments (cross-over design).

Local or national institutional Ethics Committees approved the study. Participants received the information and the opportunity to give their free and informed consent to participate in research in line with the Code of Ethics of the World Medical Association (Declaration of Helsinki), the national regulations, and the standards established by the local Institutional Review Board. All subjects underwent a screening including medical interview, physical examination, vital signs, blood chemistry and hematology tests, ECG and MRI. The subjects with chronic systemic illnesses (e.g. diabetes mellitus), receiving chronic drugs, with a history of previous or present neurological disease were excluded. They also underwent psychological interview to include subjects without psychiatric illness (no relatives with psychiatric illness), no history of alcohol and drug abuse, good sleeping habits and cognitive capacities to perform cognitive tasks.

Table 1 reports mean values ( \pm standard deviation, SD) of the demographic data in the young healthy (male) adults enrolled in the present study.

\section{2. rsEEG recordings}

The EEG signals were recorded, for at least $5 \mathrm{~min}$, from the surface of the scalp according to the International 10-20 System. A minimum of $128 \mathrm{~Hz}$ sampling frequency was used with a bandpass between $0.01 \mathrm{~Hz}$ and anti-aliasing frequency limits. It was preferred the linked-earlobe reference electrode, but not obligatory, to align with the facilities and standards of the internal protocols of the clinical recording units (i.e. Universities of Lille 2, Toulouse, and Marseille, France). Usually, a ground electrode was placed between the AFz and $\mathrm{Fz}$ electrodes, and the impedance of all electrodes was kept below $5 \mathrm{KOhm}$. Eye movements and blinks were also recorded with vertical and horizontal electro-oculographic electrodes (EOG, $0.3 \mathrm{~Hz}$ - anti-aliasing frequency limits).

In all participants, Tthe rsEEG-EOG data were recorded in the late morning (10:00-11:00 a.m.) to minimize drowsiness related to the circadian rhythm. Moreover, an operator checked on-line the subject and the rsEEG traces to keep constant the level of vigilance. Of note, the rsEEG data were collected before (Pre-sleep deprivation) and after (Post-sleep deprivation) one night of sleep deprivation (Fig. 1), immediately followed by a single dose $(100 \mathrm{mg})$ of placebo or Modafinil (pseudorandom order of the PLACEBO and MODAFINIL interventions).

\subsection{Preliminary analysis of the rSEEG data}

The following steps were performed on the rsEEG data preliminarily: (i) band-passing to avoid aliasing, (ii) down-sampling to $128 \mathrm{~Hz}$ (when recorded with higher sampling frequency), (iii) segmentation in consecutive 2-s rsEEG epochs, and (iv) off-line analysis. In case of presence of operator's markers indicating verbal

\section{Table 1}

Mean values ( \pm standard deviation, SD) of the demographic data in the young healthy (male) adults enrolled in the present study. All subjects underwent a screening including medical interview, physical examination, vital signs, blood chemistry and hematology tests, electrocardiogram (ECG) and magnetic resonance imaging (MRI) The subjects affected by chronic systemic illnesses (e.g. diabetes mellitus), receiving chronic drugs, with a history of previous or present neurological diseases were excluded. They also underwent psychological interview to include subjects without psychiatric illness, no history of alcohol and drug abuse, good sleeping habits and cognitive capacities to perform cognitive tasks. They also had no first-degree relative diagnosed with a psychiatric disorder.

\begin{tabular}{ll}
\hline & Young healthy \\
\hline $\mathrm{N}$ & 36 \\
Age (years) & $32.4 \pm 4.0 \mathrm{SD}$ \\
Education (years) & $16.9 \pm 2.1 \mathrm{SD}$ \\
Gender (Male/Female) & $36 / 0$ \\
Handedness (Right/Left) & $36 / 0$ \\
\hline
\end{tabular}




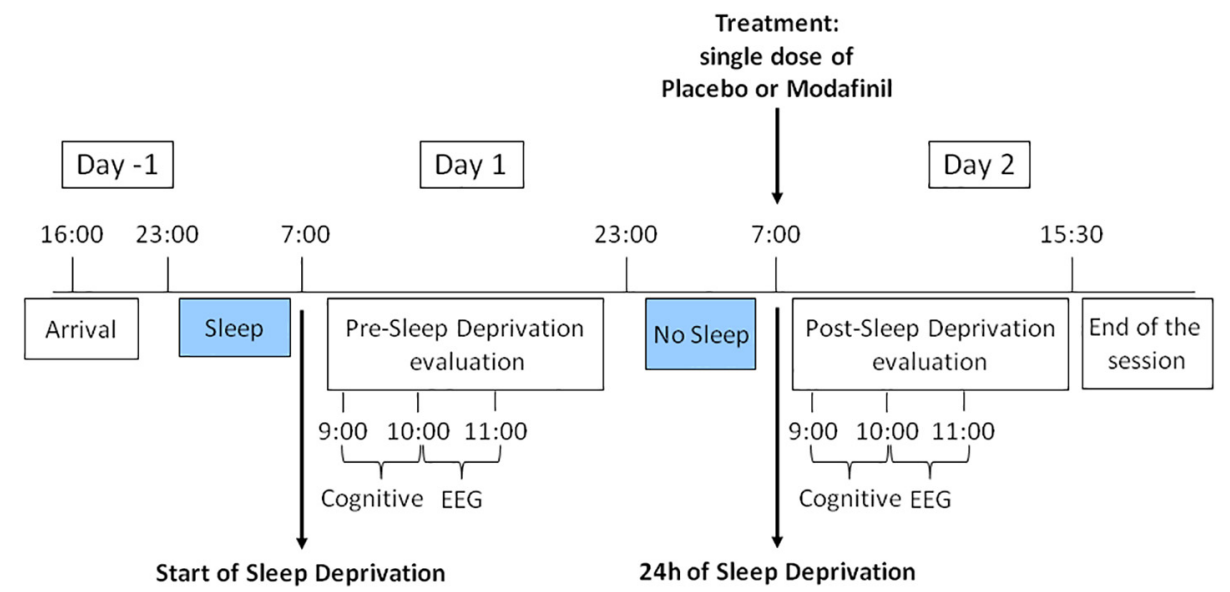

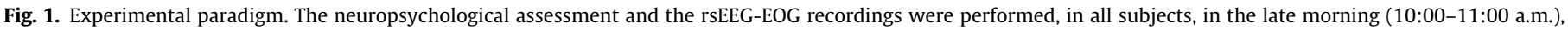

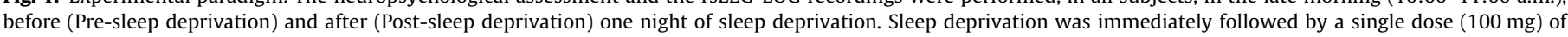
placebo or Modafinil (pseudorandom order of the PLACEBO and MODAFINIL interventions).

monitions, drowsiness, eyes movements, arm/hand actions or other disturbing events (e.g. head movements, sway, sweat), the rsEEG epochs were rejected. Moreover, the rsEEG epochs with blinking artifacts were preliminarily identified by an automatic computerized process and corrected from the EOG activity by an autoregressive method (Moretti et al., 2003). Subsequently, two independent experimenters - blind to experimental condition at the time of the rsEEG analysis - manually checked the rsEEG epochs recognized for further analysis. The rsEEG epochs with traces of a sleep interference (an on-going increase in theta, spindles, K complex) were rejected. Finally, the artifact-free rsEEG epochs were re-referenced to the common average to harmonise the rsEEG data gathered using different reference electrodes.

After the preliminary procedure for the selection of individual artifact-free rsEEG datasets, all subjects $(N=36)$ contributed to address the first working hypothesis that sleep deprivation (one night) may alter cortical sources of rsEEG rhythms in the PLACEBO condition. Instead, 33 subjects were used to address the second working hypothesis that after the sleep deprivation, a single dose of Modafinil may recover those rsEEG markers (PLACEBO vs. MODAFINIL condition). Indeed, rsEEG recordings in three individual datasets of the MODAFINIL intervention (1 Pre-sleep and 2 Postsleep deprivation) showed an insufficient number of artifact-free EEG epochs and were discharged for further analysis.

\subsection{Spectral analysis of the rSEEG data}

The power density of the rsEEG rhythms (frequency resolution: $0.5 \mathrm{~Hz}$ ) was computed with standard digital FFT-based power spectrum analysis (Hanning windowing function, Welch technique, no phase shift) using a home-made software getted under Matlab 6.5 (Mathworks Inc., Natick, MA).

Seven frequency bands of interest, used in several relevant EEG studies on dementia (Babiloni et al., 2004, 2006a, b, c, 2013a, b, c, 2014b, 2017; Besthorn et al., 1997; Chiaramonti et al., 1997; Gianotti et al., 2007; Holschneider et al., 1999; Kolev et al., 2002; Jelic et al., 1996; Leuchter et al., 1993; Nobili et al., 1999; Rodriguez et al., 1999),were considered: delta $(2-4 \mathrm{~Hz})$, theta (4-8 Hz), alpha $1(8-10.5 \mathrm{~Hz})$, alpha $2(10.5-13 \mathrm{~Hz})$, beta 1 $(13-20 \mathrm{~Hz})$, beta $2(20-30 \mathrm{~Hz})$, and gamma $(30-40 \mathrm{~Hz})$. Sharing a frequency bin by two contiguous bands is a widely accepted procedure, based on the assumption of a possible partial functional overlapping of two adjacent frequency bands.
Due to the variability of beta and gamma peaks in the power spectra of different subjects, we could not use narrow frequency bands for high-frequency bands (i.e. beta 1 , beta 2 , and gamma). For this, some limitations may affect the presented results for beta and gamma bands such as the sensitivity of EEG spectral analyses for large bands (Szava et al., 1994).

\subsection{Cortical sources of rSEEG rhythms as computed by eLORETA}

An advanced version of LORETA (low-resolution brain electromagnetic tomography; Pascual-Marqui et al., 1994) software, developed in 2007 (exact LORETA, eLORETA; Pascual-Marqui, 2007) was used to exactly localize the cortical source activity with minimum localization error, less complexity and more validation which include minimum norm. This software uses a head volume conductor model constituted by three concentric spheres: scalp, skull, and brain. In the outermost compartment (i.e. scalp), exploring electrodes can be virtually positioned to give EEG data as an input to the source estimation (Jurcak et al., 2007). The brain model is based on a realistic cerebral shape taken from a template typically used in the neuroimaging studies, namely that of the Montreal Neurological Institute (MNI152 template; Mazziotta et al., 1995). The eLORETA solves the so-called EEG inverse problem in the mentioned head volume conductor model estimating "neural" current density values at any cortical voxel for each frequency bin. The input is the EEG spectral power density calcolated at scalp electrodes. The output is the electrical brain source space formed by 6239 gray matter voxels with $5 \mathrm{~mm}$ resolution (Fuchs et al. 2002). An equivalent current dipole is placed in each voxel. For each voxel, the eLORETA package provides the Talairach coordinates, the lobe, and the Brodmann area (BA).

Afterwards, to reduce inter-subject variability and to fit EEG power density in a Gaussian distribution (Leuchter et al., 1993), this solution at each voxel (as the mean of the $\mathrm{x}, \mathrm{y}$, and $\mathrm{z}$ vectors) was normalized to the power density averaged across all the frequencies $(0.5-45 \mathrm{~Hz})$ and across all 6239 voxels of brain volume.

Due to the low spatial resolution of the present EEG methodological method (i.e. 19 scalp electrodes), the normalized eLORETA solutions were averaged within large cortical macro-regions of interest (ROIs): frontal, central, parietal, occipital, temporal, and limbic. Table 2 reports the list of the BAs used for the ROIs considered in the present study. For the present study, eLORETA solutions were estimated with a frequency resolution of $0.5 \mathrm{~Hz}$, namely, the maximum frequency resolution allowed using 2-s artefact-free 
Table 2

Regions of interest (ROIs) used for the estimation of the cortical sources of the resting state eyes-closed electroencephalographic (rsEEG) rhythms in the present study. Any ROI is defined by some Brodmann areas of the cerebral source space in the freeware used in this study, namely the exact low-resolution brain electromagnetic source tomography (eLORETA).

\begin{tabular}{ll} 
BRODMANN AREAS INTO THE REGIONS OF INTEREST (ROIs) \\
\hline Frontal & $8,9,10,11,44,45,46,47$ \\
Central & $1,2,3,4,6$ \\
Parietal & $5,7,30,39,40,43$ \\
Temporal & $20,21,22,37,38,41,42$ \\
Occipital & $17,18,19$ \\
Limbic & $31,32,33,34,35,36$
\end{tabular}

rsEEG epochs. The frequency bands of interest (i.e. from delta to gamma) were estimated as previously defined in all subjects.

\subsection{Statistical analyses}

Two statistical sessions were performed by the commercial tool STATISTICA 10 (StatSoft Inc., www.statsoft.com). In both statistical sessions, an ANOVA was performed ( $\mathrm{p}<0.05)$. The degrees of freedom were corrected by the Greenhouse-Geisser method when appropriate. Duncan test was used for post-hoc comparisons $(\mathrm{p}<0.05)$

The first statistical design tested the hypothesis that the sleep deprivation challenge would affect the cortical source activity of rsEEG rhythms. The regional normalized eLORETA current density in the PLACEBO condition was used as a dependent variable. The ANOVA factors (levels) were Time (Pre-sleep deprivation, Postsleep deprivation), Band (delta, theta, alpha 1 , alpha 2 , beta 1 , beta 2, gamma), and ROI (central, frontal, parietal, occipital, temporal, limbic). The hypothesis would be confirmed by the following two statistical results: (i) a statistical ANOVA effect including the factor Time ( $\mathrm{p}<0.05)$; (ii) a post-hoc test indicating statistically significant differences in the eLORETA cortical sources with the pattern Pre-sleep deprivation $\neq$ Post-sleep deprivation $(\mathrm{p}<0.05)$.

The second statistical design tested the hypothesis that a single dose of Modafinil would mitigate the alteration of the cortical source activity of rsEEG rhythms induced by the sleep deprivation. The difference in the regional normalized eLORETA current density between Post-sleep deprivation and Pre-sleep deprivation (Postsleep deprivation minus Pre-sleep deprivation) was used as an input. The ANOVA factors (levels) were Condition (PLACEBO, MODAFINIL), Band (delta, theta, alpha 1, alpha 2, beta 1, beta 2, gamma), and ROI (central, frontal, parietal, occipital, temporal, limbic). The hypothesis would be confirmed by the following two statistical results: (i) a statistical ANOVA effect including the factor Condition $(\mathrm{p}<0.05)$; (ii) a post-hoc test indicating statistically significant differences in the eLORETA cortical sources with the pattern PLACEBO $\neq$ MODAFINIL $(\mathrm{p}<0.05)$.

\section{Results}

Fig. 2 illustrates the grand average of the normalized eLORETA solutions (i.e. normalized dipole current density at cortical voxels) modeling the activity of distributed EEG cortical sources for (i) two conditions (PLACEBO, MODAFINIL), (ii) two times (Pre-sleep deprivation, Post-sleep deprivation), and (iii) seven bands (delta, theta, alpha 1 , alpha 2 , beta 1 , beta 2 , and gamma). In both the conditions (PLACEBO, MODAFINIL), the Pre-sleep deprivation period was characterized by delta sources with a widespread moderate activity, and alpha 1 sources with the maximal activity distributed in parietal, occipital, and temporal regions. Theta and alpha 2 sources showed a moderate activity when compared to that of alpha 1 sources. Finally, beta 1 , beta 2 , and gamma sources were characterized by lowest activity. In both conditions (PLACEBO, MODAFINIL), the alpha 1 source activity was lower in the Post- than Pre-Sleep deprivation. This effect was greater in the PLACEBO than the MODAFINIL condition as a possible beneficial impact of the Modafinil over placebo. Finally, we reported that in the PLACEBO (but not MODAFINIL) condition, the delta source activity was greater in the Post- than Pre-Sleep deprivation as another possible index of the beneficial effect of the Modafinil.

Fig. 3 shows the mean values $( \pm$ SE) of the eLORETA cortical source activity of the rsEEG rhythms for the following factors: (i) Time (Pre-sleep deprivation, Post-sleep deprivation; PLACEBO condition), (ii) Band (delta, theta, alpha 1, alpha 2, beta 1, beta 2, gamma), and (iii) ROI (central frontal, parietal, occipital, temporal, limbic). Notably, the rsEEG source activity differed across various cortical macro regions, thus supporting the idea that scalp rsEEG rhythms are generated by a distributed pattern of cortical sources. The ANOVA showed a statistically significant interaction effect of the factors Time, Band, and ROI $(\mathrm{F}(30,1050)=6.5, \mathrm{p}<0.00001)$. Duncan post-hoc test $(\mathrm{p}<0.05)$ provided the following results: (i) the eLORETA cortical source pattern Post-sleep deprivation $>$ Presleep deprivation time was fitted by parietal delta sources $(\mathrm{p}=0.01)$; (ii) the eLORETA cortical source pattern Post-sleep deprivation < Pre-sleep deprivation time was fitted by central $(p=0.000001)$, parietal $(p=0.00001)$, occipital $(p=0.00001)$, temporal $(p=0.000001)$, and limbic $(p=0.000005)$ alpha 1 sources as well as parietal $(p=0.00001)$, occipital $(p=0.000005)$, and limbic $(p=0.05)$ alpha 2 sources. Furthermore, the effect sizes (Cohen's d) were calculated for the above nine mentioned LORETA solutions presented statistically significant pattern Post-sleep deprivation $\neq$ Pre-sleep deprivation time. The effect sizes (Cohen's d) provided the following results: 0.28 for parietal delta, -0.43 for central alpha $1,-0.47$ for parietal alpha $1,-0.47$ for occipital alpha $1,-0.43$ for temporal alpha $1,-0.50$ for limbic alpha $1,-0.15$ for parietal alpha $2,-0.24$ for occipital alpha 2 , and -0.25 for limbic alpha 2 sources.

These results suggest that the sleep deprivation challenge affected the activity in the eLORETA cortical sources of rsEEG rhythms in the healthy young volunteers.

Fig. 4 shows the mean values $( \pm S E)$ of the eLORETA cortical source activity in the rsEEG rhythms when that activity is subtracted between the Post-sleep deprivation and the Pre-sleep deprivation time (Post-sleep deprivation minus Pre-sleep deprivation). These mean values are illustrated for the following factors: (i) Condition (PLACEBO, MODAFINIL), (ii) Band (delta, theta, alpha 1 , alpha 2 , beta 1 , beta 2 , gamma), and (iii) ROI (central frontal, parietal, occipital, temporal, limbic). In the figure, zero values mean the same eLORETA cortical source activity in the Pre-sleep deprivation and the Post-sleep deprivation time. Negative values in this subtraction mean a lower eLORETA cortical source activity in the Post-sleep deprivation than the Pre-sleep deprivation time. Vice versa for the positive values. The higher the positive values at the delta band, the higher the interference effect of the sleep deprivation. The higher the negative values at the alpha band, the higher the interference effect of the sleep deprivation. Notably, the rsEEG source activity differed across various cortical macro regions, thus supporting the idea that scalp rsEEG rhythms are generated by a distributed pattern of cortical sources. The ANOVA showed a statistically significant interaction effect of the factors Condition, Band, and ROI $(\mathrm{F}(30,960)=2.9, \mathrm{p}<0.0001)$. Duncan post-hoc test $(p<0.05)$ unveiled the following 6 cortical delta and alpha sources with significant effects: (i) the eLORETA source pattern MODAFINIL < PLACEBO was fitted by the parietal $(p=0.00003)$ and occipital $(p=0.005)$ delta sources; (ii) the eLORETA source pattern MODAFINIL > PLACEBO was fitted by the parietal $(p=0.00001)$ and occipital $(p=0.00002)$ alpha 1 sources as 


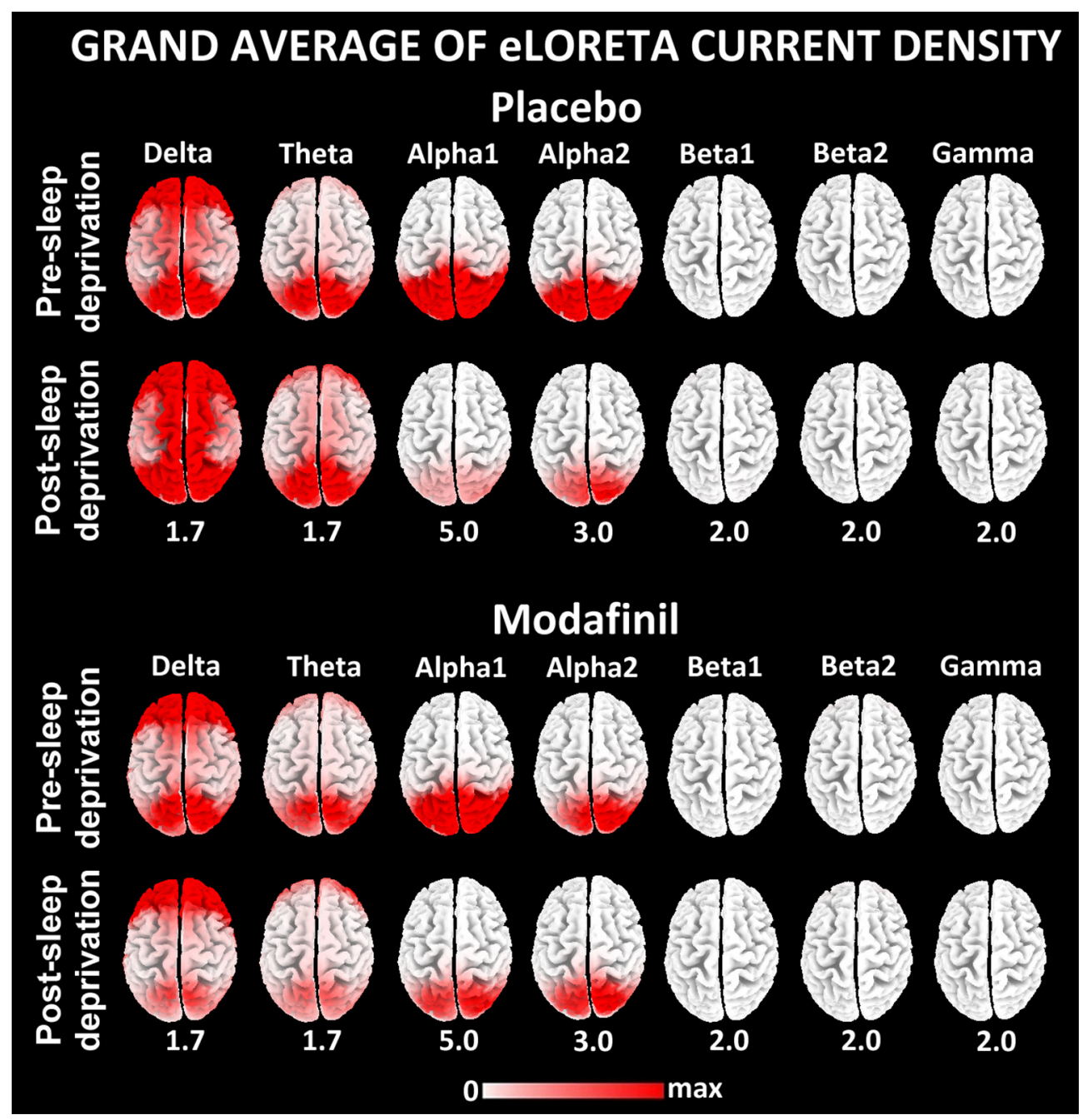

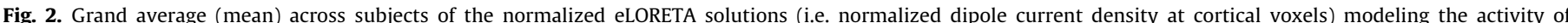

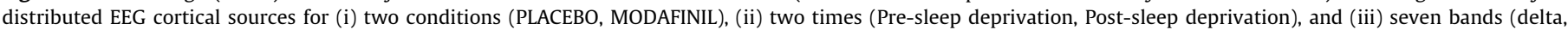

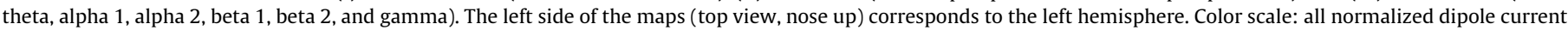

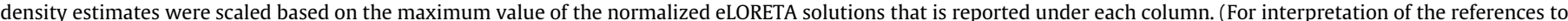
color in this figure legend, the reader is referred to the web version of this article.)

well as the parietal $(\mathrm{p}=0.005)$ and occipital $(\mathrm{p}=0.002)$ alpha 2 sources. Furthermore, the effect sizes (Cohen's d) were calculated for the above six mentioned LORETA solutions presented statistically significant pattern MODAFINIL $\neq$ PLACEBO. The effect sizes (Cohen's d) provided the following results: 0.46 for parietal delta, 0.43 for occipital delta, -0.20 for parietal alpha $1,-0.18$ for occipital alpha $1,-0.18$ for parietal alpha 2 , and -0.22 for occipital alpha 2 sources.

These 6 delta and alpha sources were used as an input for the subsequent analysis with mixed linear models $(\mathrm{p}<0.05)$, to test the hypothesis of an interdependence between these sources and a composite measure of subjects' cognitive performances. The composite measure was obtained as follows. Firstly, the individual scores of six neuropsychological tests (i.e., Rey Auditory Verbal Learning Test Learning phase, Rey Auditory Verbal Learning Recall phase, Semantic verbal fluency task, Phonemic fluency task, Digit Span Forward, and Digit Span backward) were considered. For any single test, the original scores of all subjects in the pre- and post-sleep deprivation phases and the PLACEBO and MODAFINIL conditions formed a distribution of values. In this distribution, the original scores were rescaled to assume minimum and maximum values equal to 0 and 1 , respectively, while the other scores assumed rescaled values from 0 to 1 proportionally. As an outcome, for that single test any subject was associated with 4 rescaled values (i.e., PLACEBO Pre-sleep deprivation, PLACEBO Post-sleep deprivation, MODAFINIL Pre-sleep deprivation, and MODAFINIL Post-sleep deprivation). This operation was repeated for all 6 neuropsychological tests. Secondly, the global composite cognitive measure was calculated for a given subject, averaging the rescaled values of 6 neuropsychological tests. As an outcome, any subject was associated with 4 global composite cognitive measures (i.e., PLACEBO Pre-sleep deprivation, PLACEBO Post-sleep deprivation, MODAFINIL Pre-sleep deprivation, and MODAFINIL Post-sleep deprivation). Concerning the linear mixed models $(\mathrm{p}<0.05)$, the mentioned 6 (eLORETA) cortical sources were considered as independent variables, while Condition (PLACEBO, MODAFINIL) and Time (Pre-sleep deprivation, Post-sleep deprivation) were used as factors. In total, 6 linear mixed models were performed ( $p<0.05$ ), one for any significant delta or alpha cortical source. The results only showed a statistically significant main effect for the occipital $(\mathrm{F}=9.9, \mathrm{p}=0.002)$ alpha 2 source activities, thus confirming a strict covariance between those posterior alpha source activities during the experiments and subjects' global cognitive performances. 


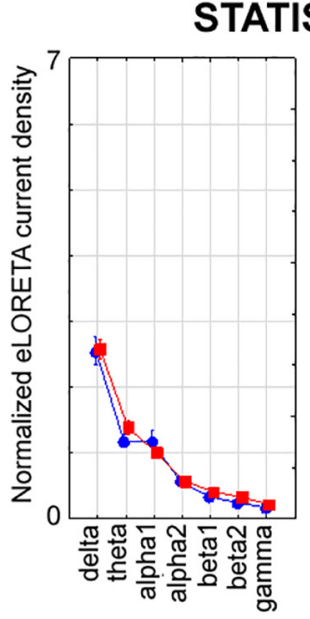

Frontal

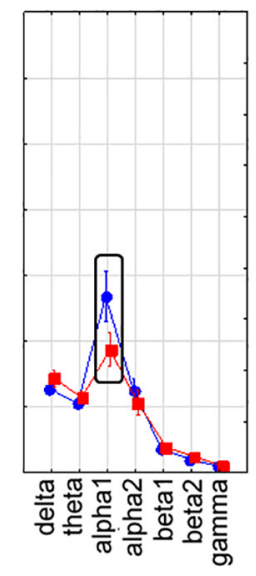

Central

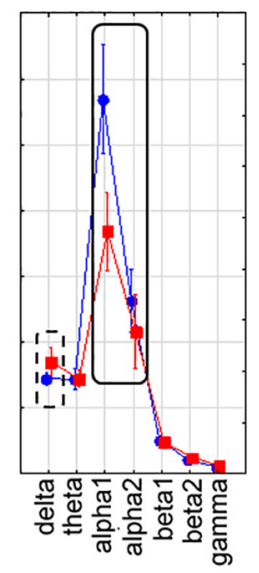

Parietal

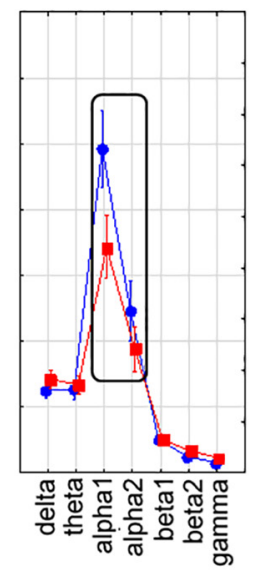

Occipital

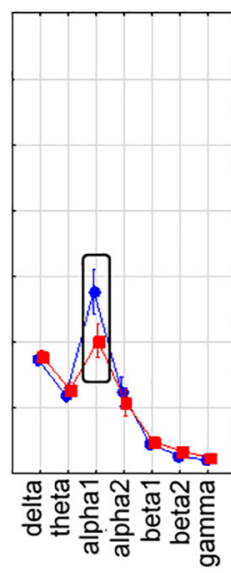

Temporal

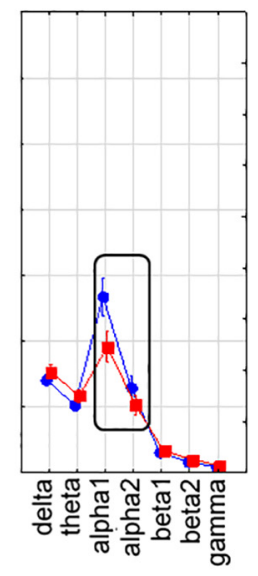

Limbic
Pre-sleep deprivation

Post-sleep deprivation
Post-sleep deprivation $<$ Pre-sleep deprivation $(p<0.05)$

I_-_; Post-sleep deprivation $>$ Pre-sleep deprivation $(p<0.05)$

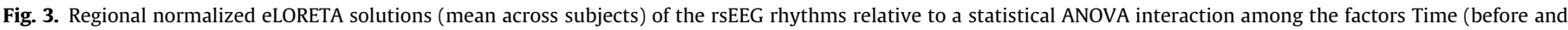

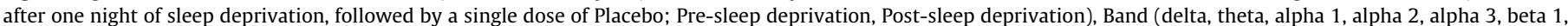

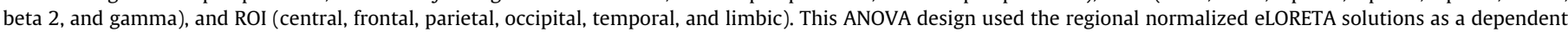

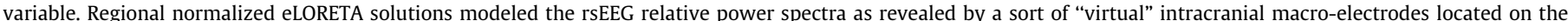

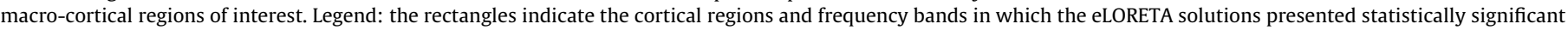
eLORETA pattern: Pre-sleep deprivation $\neq$ Post-sleep deprivation $(p<0.05)$; the variability bars indicate the standard error of the mean, SE.

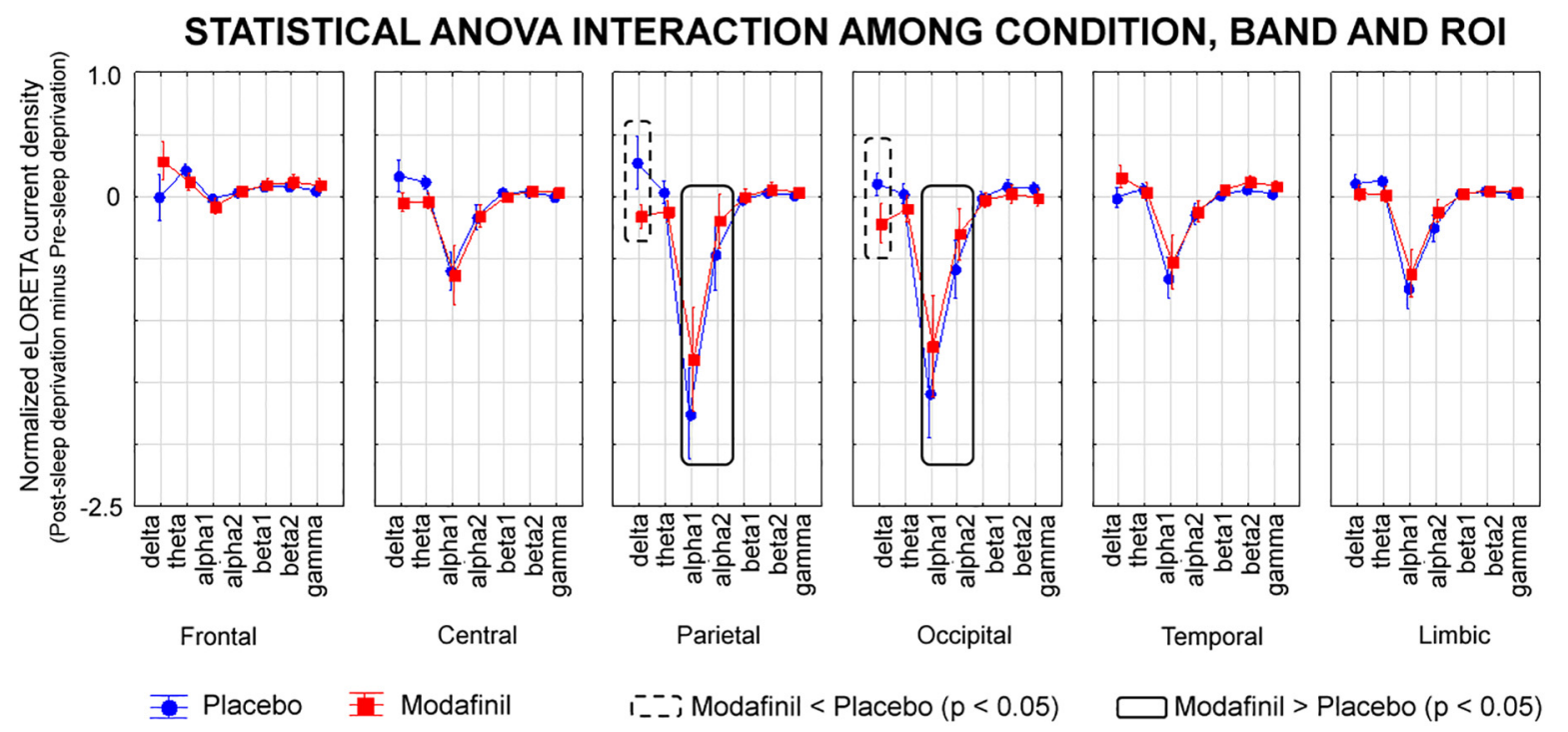

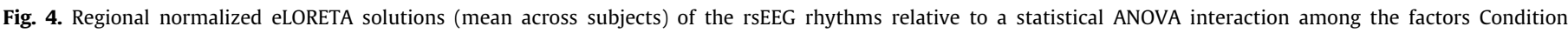

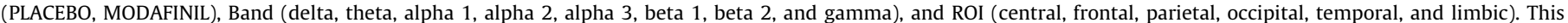

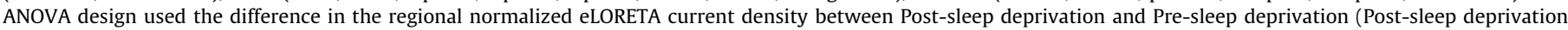

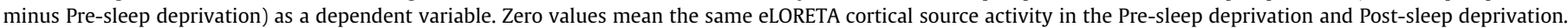

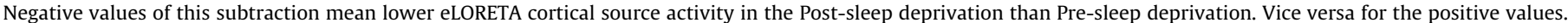

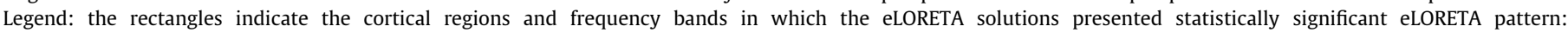
PLACEBO $\neq$ MODAFINIL $(p<0.05)$; the variability bars indicate the SE.

A control ANOVA $(\mathrm{p}<0.05)$ evaluated the effects of sleep deprivation and Modafinil on subjects' global cognitive performances as revealed by the mentioned global composite cognitive measure (dependent variable). The ANOVA factors were Condition (PLACEBO, MODAFINIL; independent variable) and Time (Pre-sleep deprivation, Post-sleep deprivation). Fig. 5 shows the mean values
$( \pm \mathrm{SE})$ of the global composite cognitive measure (arcsine square root transformed) in the healthy young adults $(N=36)$ for the two conditions (PLACEBO, MODAFINIL) and the two times (Presleep deprivation, Post-sleep deprivation) of the experiments. The ANOVA results showed no statistically significant effects on the dependent variable $(p>0.05)$, thus indicating that the sleep 


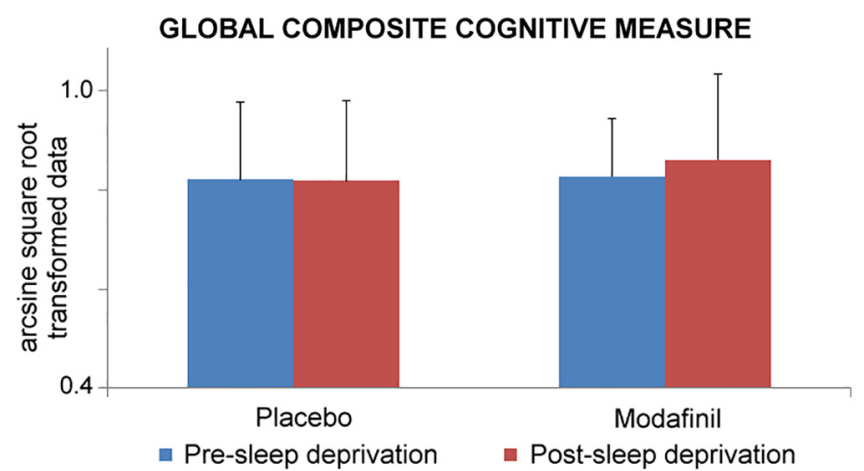

Fig. 5. Mean values ( \pm standard deviation, SD) of the global composite cognitive measure (arcsine square root transformed) in the healthy young adults $(N=36)$ for the two conditions (PLACEBO, MODAFINIL) and the two times (Pre-sleep deprivation, Post-sleep deprivation) of the experiments.

deprivation and Modafinil did not affect the subjects' global composite cognitive measure.

\section{Discussion}

In the present study of the PharmaCog project, we tested the hypothesis that one night of sleep deprivation and a single dose of a drug enhancing vigilance (i.e. Modafinil) may affect posterior cortical sources of delta and alpha rhythms in healthy young adults resting in relaxed wakefulness, thus suggesting a strict relationship between those rsEEG source activities and physiological/pharmacological modulators of human vigilance in quiet wakefulness.

The present results showed that the sleep deprivation induced an increase in the parietal delta source activity and a decrease in the posterior (i.e. parietal, occipital, temporal and limbic) alpha 1 and alpha 2 source activities. These findings suggest that sleep deprivation is able to deteriorate posterior cortical delta and alpha sources, possibly reflecting neurophysiological mechanisms regulating brain arousal during the quiet vigilance (Babiloni et al., 2014a).

The present results also showed that compared to the placebo, a single dose of a vigilance enhancer (i.e., Modafinil) - administered immediately after the sleep deprivation - induced a significant recovery of the mentioned posterior cortical delta and alpha source activities, some of them related to subjects' global cognitive performances probed by standard neuropsychological tests. These results extend to the regional cortical source space previous rsEEG evidence in the literature (Cajochen et al., 1995; Dumont et al., 1999; Corsi-Cabrera et al., 1996; Mander et al., 2010). Previous studies have reported that compared to a placebo, a single dose of Modafinil (200 mg) during 1 night of sleep deprivation partially recovered rsEEG rhythms recorded over the whole scalp in healthy (male) young adults; this "global" effect was described at delta, theta, alpha, and beta bands (James et al., 2011). Furthermore, 3 doses of Modafinil (300 mg) before and during a prolonged sleep deprivation $(60 \mathrm{~h})$ partially recovered the alteration of global scalp rsEEG rhythms at the alpha band in healthy young adults (Chapotot et al., 2003). Moreover, these alpha rhythms were less deranged after sleep deprivation and were more sensitive to Modafinil dosages (two single doses, $100 \mathrm{mg}$, during sleep deprivation) in healthy (male) young adults with Val/Val than Met/Met allele carriers (Bodenmann et al., 2009). Finally, the chronic administration of Modafinil (100 mg and $400 \mathrm{mg}$ ) for 3 weeks partially recovered the alteration in prefrontal, parietal, and temporal rsEEG source activities at the alpha band in drug-free patients with narcolepsy (Saletu et al., 2004, 2007). This effect was related to an improvement in cognitive performances (Saletu et al., 2007) and scores of Multiple Sleep Latency Test and Epworth Sleepiness Scale (Saletu et al., 2004).

In relation to those previous investigations, the present study unveiled significant effects of a moderate dose (100 mg) of Modafinil on posterior cortical delta and alpha source activities in healthy (male) young adults after just 1 night of sleep deprivation. Instead, the score of standard neuropsychological tests evaluating cognitive functions were not affected by sleep deprivation and Modafinil acute administration. Overall, these results confirm the strict relationship between these delta and alpha source activities and physiological and pharmacological manipulations of the vigilance in the current healthy young adults (This theoretical conclusion is valid even if this study was conducted only in male subjects). Furthermore, results suggest that healthy young adults can arouse brain activity and vigilance to ensure a good cognitive performance in the neuropsychological assessment even after a sleep deprivation night. This conclusion emphasizes the need of biomarkers probing brain arousal in quiet vigilance to complement standard neuropsychological exams in the assessment of human higher functions.

Interestingly, the cortical source estimation of the present approach allowed testing the hypothesis that the sleep deprivation effects in healthy volunteers were reminiscent of the alteration of the rsEEG sources investigated in patients with ADD and its prodromal stage of $\mathrm{ADMCl}$. The present results globally confirmed that hypothesis in the light of the following previous findings obtained with the same rsEEG source estimation in the occipital and parietal cortex: (i) these cortical sources of alpha rhythms (about 8-10 Hz) were abnormal in ADD patients when compared to ADMCI, cerebrovascular dementia, and Parkinson's disease subjects (Babiloni et al., 2004, 2006a, 2017); (ii) those of delta (<4 Hz) and/or alpha rhythms were related to the global cognitive status, brain amyloidosis and neurodegeneration, and genetic risk factors for ADD and ADMCI patients (Babiloni et al., 2006b,c, 2013a; Galluzzi et al., 2016); and (iii) the cortical sources of delta and alpha rhythms deteriorated across time (1 year) in ADD and ADMCI patients (Babiloni et al., 2013b, 2014b). Overall, posterior cortical delta and alpha sources exhibited congruent alterations in the present healthy adults after sleep deprivation and the $\mathrm{AD}$ patients in previous PharmaCog studies (Babiloni et al., 2010, 2013; Galluzzi et al., 2016; Jovicich et al., 2019), namely increased delta and decreased alpha sources. Furthermore, those alpha sources were related to scores of neuropsychological tests in AD patients (Babiloni et al., 2010, 2013; Galluzzi et al., 2016; Jovicich et al., 2019) and the present healthy adults. Therefore, neurophysiological mechanisms underlying quiet vigilance may be affected in both AD patients and healthy young adults after one night of sleep deprivation. However, this conclusion should be considered as preliminary as healthy young adults and AD patients show obvious differences in the integrity of the brain structure and the availability of synaptic contacts/excitatory neurotransmitters (e.g., acetylcholine and serotonin) modulating vigilance and attention in frontoparietal, thalamus-cortical, and ascending reticular activating systems involving hypothalamus and basal ganglia.

The neurophysiological mechanisms underlying these related effects of the sleep deprivation and Modafinil on the rsEEG rhythms in the present healthy adults remain unclear. At this early stage of the research, we speculate that both sleep deprivation and AD processes might downregulate thalamus-cortical mechanisms generating posterior cortical delta and alpha rhythms. On one hand, sleep deprivation might be associated with a progressive reduction in signaling from thalamocortical high-threshold neurons to GABAergic thalamic interneurons and thalamocortical relay-mode neurons, the latter being the trigger of cortical pyramidal neurons generating cortical alpha source activities (Hughes and Crunelli, 2005; Lörincz et al., 2008, 2009; Lopes da Silva, 2013). 
Furthermore, thalamocortical neural activities may partially switch from tonic to burst mode entraining oscillatory signals at delta frequencies in that brain circuit (Hughes and Crunelli, 2005; Lörincz et al., 2008, 2009). As a result, posterior cortical alpha rhythms might switch to theta frequencies and delta rhythms in a gray zone between quiet wakefulness and sleep onset (Hughes and Crunelli, 2005; Lörincz et al., 2008, 2009).

On the other hand, AD neuropathology (especially insoluble Aß42) may diffusely desynchronize the mentioned thalamuscortical circuit with an effect of "overexcitation". In this line, posterior cortical alpha rhythms might desynchronize, and slower EEG waves at delta frequencies might appear. Part of these waves might be considered as mild manifestations of subclinical, nonconvulsive, epileptiform EEG activities described in some AD patients (Horváth et al., 2016; Scarmeas et al., 2009; Vossel et al., 2013).

Several ascending activating systems may regulate the above thalamus-cortical circuit. Among them, cholinergic neuromodulatory systems may play a prominent role as demonstrated by the following previous findings. Firstly, cholinergic basal forebrain retransmit noradrenergic (locus coeruleus) and glutamatergic (brainstem reticular formation) signals arousing limbic and thalamus-cortical circuits (Jones, 2004) and desynchronizes cortical EEG rhythms inducing beta $(13-30 \mathrm{~Hz})$ and gamma $(>30 \mathrm{~Hz})$ oscillatory activities (Kalmbach et al., 2012; Bohnen et al., 2018). Secondly, a single dose of a muscarinic cholinergic antagonist (i.e. scopolamine) transiently increased resting state cortical delta and theta rhythms in healthy adults, while it reduced alpha and beta rhythms (Ebert and Kirch, 1998; Liem-Moolenaar et al., 2011). Thirdly, a similar effect was observed in both healthy subjects and $\mathrm{AD}$ patients as a function of the integrity of cholinergic neurotransmission (Neufeld et al., 1994). Fourthly, a single dose of scopolamine deranged delta to gamma rhythms in ADD patients resting in quiet wakefulness (Johannsson et al., 2015; Snaedal et al., 2010). Fifthly, a single dose of Acetylcholinesterase inhibitors enhanced the cortical alpha-theta ratio in $\mathrm{AD}$ patients clinically responding to a long chronic treatment with that drug (Alhainen et al., 1991). Sixthly, long chronic treatment with Acetylcholinesterase inhibitors had beneficial effects on posterior cortical alpha rhythms in ADD patients in some studies (Babiloni et al., 2006d; Balkan et al., 2003). In other studies, those beneficial effects were observed in cortical delta (Balkan et al., 2003; Gianotti et al., 2008; Brassen and Adler, 2003) and theta (Brassen and Adler, 2003; Gianotti et al., 2008) rhythms.

The present results do not grant that Modafinil administration in $\mathrm{AD}$ patients can induce beneficial clinical effects on vigilance and, consequently, cognitive processes. We can just speculate that the present and some previous data make this hypothesis quite promising. In previous studies, Modafinil showed the following action mechanisms (Chemelli et al., 1999; Gerrard and Malcolm, 2007; Lin et al., 1996): (i) increase in cortical cellular creatinephosphocreatine pool and several excitatory aminergic neurotransmitters in the synaptic cleft such as dopamine and noradrenaline; (ii) reduction in cortical GABA inhibitory neurotransmitters by serotoninergic mediated pathways in brain regions modulating brain arousal; and (iii) increase in the activity in anterior hypothalamus and surrounding areas modulating brain arousal possibly via orexin neurons. To test that promising hypothesis, future investigations may bioethically adapt the present experiments to $\mathrm{AD}$ research. For example, Nold and preclinical $\mathrm{AD}$ (e.g., subjective memory complaint and positivity to cerebrospinal markers of AD) subjects may undergo to a sleep deprivation lasting only few hours before the administration of Modafinil or placebo.

In the present study, $128-\mathrm{Hz}$ sampling rate was used for the present rsEEG data analysis, as it maximized the inclusion of individual rsEEG datasets, allowing us to fit the estimated sample size.
As mentioned above, the working hypothesis of this study focused on posterior cortical delta $(2-4 \mathrm{~Hz})$ and alpha $(8-13 \mathrm{~Hz})$ source activities computed from rsEEG rhythms, based on previous findings of our Consortium in AD patients (Babiloni et al., 2004, 2006a, b, c, 2013a, b, c, 2014b, 2017; Galluzzi et al., 2016). Therefore, the use of $128-\mathrm{Hz}$ sampling rate for the present rsEEG data analysis did not affect the novel findings of this study.

Concerning the gamma band, the present Figs. 2-4 unveiled that (eLORETA) source activities at beta 2 and gamma (i.e., $20-40 \mathrm{~Hz}$ ) bands were negligible in the current experimental conditions (i.e. eyes-closed resting state, sleep deprivation, Modafinil acute dose). However, the present $128-\mathrm{Hz}$ sampling frequency did not allow us the spectral analysis of rsEEG signals $>40 \mathrm{~Hz}$, for the risk of some distortions of low-band frequencies (i.e., the aliasing issue). Therefore, future studies using a spatial sampling of EEG activity $>256 \mathrm{~Hz}$ or higher should be performed for extending the present investigations to high-frequency gamma bands $>40 \mathrm{~Hz}$.

\section{Conclusions}

The present study of the PharmaCog project report that the sleep deprivation induced an increase in posterior cortical delta source activities and a reduction in widespread alpha source activities, while the acute administration of Modafinil partially recovered these effects.

These results have two main neurophysiological implications. Firstly, the mentioned effects of sleep deprivation and Modafinil suggest that in quiet wakefulness, not only alpha but also delta cortical rhythms in posterior areas may reflect neurophysiological oscillatory mechanisms underlying quiet vigilance in healthy adults. Therefore, posterior cortical delta rhythms should be considered neither as (i) a mere epiphenomenon in the human brain resting in quiet wakefulness and nor (ii) only a reflection of brain abnormalities in patients with neurological disorders such as AD.

Secondly, the present results may enrich the interpretation of abnormal posterior cortical delta and alpha source activities reported in $A D$ patients in the mentioned PharmaCog rsEEG studies. It can be speculated that in AD patients, alterations in both posterior cortical delta and alpha source activities may reflect the interaction of $\mathrm{AD}$ neuropathological processes on neurophysiological oscillatory mechanisms underlying brain arousal and vigilance in quiet wakefulness. If confirmed in future cross-validation studies carried out in healthy young adults, those delta and alpha source activities may be used as surrogate endpoints of those mechanisms for the evaluation of drug candidates designed to normalize brain arousal and vigilance in AD patients.

\section{Declaration of Competing Interest}

None of the authors have potential conflicts of interest to be disclosed.

\section{Acknowledgements}

The research leading to the current results has received funding from the European Community's Seventh Framework Programme (FP7/2007-2013) for the Innovative Medicine Initiative under grant agreement $n^{\circ} 115009$ (Prediction of cognitive properties of new drug candidates for neurodegenerative diseases in early clinical development, PharmaCog).

\section{References}

Achermann P. The two-process model of sleep regulation revisited. Aviat Space Environ Med. 2004;75:A37-43. 
Aeschbach D, Matthews JR, Postolache TT, Jackson MA, Giesen HA, Wehr TA Dynamics of the human EEG during prolonged wakefulness: evidence for frequency-specific circadian and homeostatic influences. Neurosci Lett 1997;239(2-3):121-4.

Alhainen K, Partanen J, Reinikainen K, Laulumaa V, Soininen H, Airaksinen M, et al. Discrimination of tetrahydroaminoacridine responders by a single dose pharmaco-EEG in patients with Alzheimer's disease. Neurosci Lett 1991;127 (1):113-6.

Babiloni C, Binetti G, Cassetta E, Cerboneschi D, Dal Forno G, Del Percio C, et al Mapping distributed sources of cortical rhythms in mild Alzheimers disease. A multi-centric EEG study. Neuroimage 2004;22:57-67.

Babiloni C, Binetti G, Cassetta E, Dal Forno G, Del Percio C, Ferreri F, et al. Sources of cortical rhythms change as a function of cognitive impairment in pathological aging: a multi-centric study. Clin Neurophysiol 2006a;117:252-68.

Babiloni C, Benussi L, Binetti G, Bosco P, Busonero G, Cesaretti S, et al. Genotype (cystatin C) and EEG phenotype in Alzheimer disease and mild cognitive impairment: a multicentric study. Neuroimage 2006b;29(3):948-64.

Babiloni C, Benussi L, Binetti G, Cassetta E, Dal Forno G, Del Percio C, et al. Apolipoprotein E and alpha brain rhythms in mild cognitive impairment: multicentric EEG study. Ann Neurol 2006c;59:323-34.

Babiloni C, Cassetta E, Dal Forno G, Del Percio C, Ferreri F, Ferri R, et al. Donepezil effects on sources of cortical rhythms in mild Alzheimer's disease: responders vs non-responders. Neuroimage 2006d;31(4):1650-65.

Babiloni C, Lizio R, Vecchio F, Frisoni GB, Pievani M, Geroldi C, et al. Reactivity of cortical alpha rhythms to eye opening in mild cognitive impairment and Alzheimer's disease: an EEG study. J Alzheimers Dis 2010;22(4):1047-64. https://doi.org/10.3233/JAD-2010-100798.

Babiloni C, Del Percio C, Bordet R, Bourriez JL, Bentivoglio M, Payoux P, et al. Effects of acetylcholinesterase inhibitors and memantine on resting-state electroencephalographic rhythms in Alzheimer's disease patients. Clin $\begin{array}{lll}\text { Neurophysiol 2013a;124(5):837-50. https://doi.org/10.1016/ } & \text { 20 }\end{array}$ j.clinph.2012.09.017.

Babiloni C, Carducci F, Lizio R, Vecchio F, Baglieri A, Bernardini S, et al. Resting state cortical electroencephalographic rhythms are related to gray matter volume in subjects with mild cognitive impairment and Alzheimer's disease. Hum Brain Mapp 2013b;34:1427-46.

Babiloni C, Lizio R, Del Percio C, Marzano N, Soricelli A, Salvatore E, et al. Cortical sources of resting state EEG rhythms are sensitive to the progression of early stage Alzheimer's disease. J Alzheimers Dis 2013c;34(4):1015-35.

Babiloni C, Del Percio C, Lizio R, Infarinato F, Blin O, Bartres-Faz D, et al. A review of the effects of hypoxia, sleep deprivation and transcranial magnetic stimulation on EEG activity in humans: challenges for drug discovery for Alzheimer's disease. Curr Alzheimer Res 2014a;11(5):501-18.

Babiloni C, Del Percio C, Lizio R, Marzano N, Infarinato F, Soricelli A, et al. Cortical sources of resting state electroencephalographic alpha rhythms deteriorate across time in subjects with amnesic mild cognitive impairment. Neurobiol Aging 2014b;35(1):130-42.

Babiloni C, Del Percio C, Lizio R, Noce G, Cordone S, Lopez S, et al. Abnormalities of cortical neural synchronization mechanisms in patients with dementia due to Alzheimer's and Lewy body diseases: an EEG study. Neurobiol Aging 2017;55:143-58. https://doi.org/10.1016/i.neurobiolaging.2017.03.030.

Balkan S, Yaraş N, Mihçi E, Dora B, Ağar A, Yargiçoğlu P. Effect of donepezil on EEC spectral analysis in Alzheimer's disease. Acta Neurol Belg 2003;103(3):164-9.

Besthorn C, Zerfass R, Geiger-Kabisch C, Sattel H, Daniel S, Schreiter-Gasser U, et al. Discrimination of Alzheimer's disease and normal aging by EEG data. Electroencephalogr Clin Neurophysiol 1997;103(2):241-8.

Bodenmann S, Rusterholz T, Dürr R, Stoll C, Bachmann V, Geissler E, et al. The functional Val158Met polymorphism of COMT predicts interindividual differences in brain alpha oscillations in young men. J Neurosci 2009;29 (35):10855-62.

Bohnen NI, Grothe MJ, Ray NJ, Müller MLTM, Teipel SJ. Recent advances in cholinergic imaging and cognitive decline-Revisiting the cholinergic hypothesis of dementia. Curr Geriatr Rep 2018;7(1):1-11.

Bonanni L, Thomas A, Tiraboschi P, Perfetti B, Varanese S, Onofrj M. EEG comparisons in early Alzheimer's disease, dementia with Lewy bodies and Parkinson's disease with dementia patients with a 2-year follow-up. Brain 2008;131:690-705.

Brassen S, Adler G. Short-term effects of acetylcholinesterase inhibitor treatment on EEG and memory performance in Alzheimer patients: an open, controlled trial. Pharmacopsychiatry 2003;36(6):304-8.

Breslau J, Starr A, Sicotte N, Higa J, Buchsbaum MS. Topographic EEG changes with normal aging and SDAT. Electroencephalogr Clin Neurophysiol 1989;72 (4):281-9.

Briel RC, McKeith IG, Barker WA, Hewitt Y, Perry RH, Ince PG, et al. EEG findings in dementia with Lewy bodies and Alzheimer's disease. J Neurol Neurosuro Psychiatry 1999;66(3):401-3.

Cajochen C, Brunner DP, Kräuchi K, Graw P, Wirz-Justice A. Power density in theta/ alpha frequencies of the waking EEG progressively increases during sustained wakefulness. Sleep 1995;18(10):890-4.

Cajochen C, Knoblauch V, Kräuchi K, Renz C, Wirz-Justice A. Dynamics of frontal EEC activity, sleepiness and body temperature under high and low sleep pressure. Neuroreport 2001:12(10):2277-81.

Cassé-Perrot C, Lanteaume L, Deguil J, Bordet R, Auffret A, Otten L, et al. Neurobehavioral and cognitive changes induced by sleep deprivation in healthy volunteers. CNS Neurol Disord Drug Targets 2016;15(7):777-801.
Chapotot F, Pigeau R, Canini F, Bourdon L, Buguet A. Distinctive effects of modafinil and $\mathrm{d}$-amphetamine on the homeostatic and circadian modulation of the human waking EEG. Psychopharmacology (Berl) 2003;166(2):127-38.

Chemelli RM, Willie JT, Sinton CM, Elmquist JK, Scammell T, Lee C, et al. Narcolepsy in orexin knockout mice: molecular genetics of sleep regulation. Cell 1999;98 (4):437-51.

Chiaramonti R, Muscas GC, Paganini M, Müller TJ, Fallgatter AJ, Versari A, et al. Correlations of topographical EEG features with clinical severity in mild and moderate dementia of Alzheimer type. Neuropsychobiology 1997·36(3):153-8.

Corsi-Cabrera M, Arce C, Ramos J, Lorenzo I, Guevara MA. Time course of reaction time and EEG while performing a vigilance task during total sleep deprivation. Sleep 1996;19(7):563-9.

Corsi-Cabrera M, Sánchez AI, del-Río-Portilla Y, Villanueva Y, Pérez-Garci E. Effect of $38 \mathrm{~h}$ of total sleep deprivation on the waking EEG in women: sex differences. Int J Psychophysiol 2003;50(3):213-24.

Daulatzai MA. Early stages of pathogenesis in memory impairment during normal senescence and Alzheimer's disease. J Alzheimers Dis. 2010;20:355-67.

Dierks T, Ihl R, Frolich L, Maurer K. Dementia of the Alzheimer type: effects on the spontaneous EEG described by dipole sources. Psychiatry Res 1993;50:151-62.

Dubois B, Feldman HH, Jacova C, Hampel H, Molinuevo JL, Blennow K, et al. Advancing research diagnostic criteria for Alzheimer's disease: the IWG-2 criteria. Lancet Neurol 2014;13(6):614-29.

Dumont M. Macchi MM, Carrier J, Lafrance C, Hébert M. Time course of narrow frequency bands in the waking EEG during sleep deprivation. Neuroreport 1999; $10(2): 403-7$

Ebert U, Kirch W. Scopolamine model of dementia: electroencephalogram findings and cognitive performance. Eur J Clin Invest 1998;28(11):944-9.

Ebert U, Grossmann M, Oertel R, Gramatté T, Kirch W. Pharmacokineticpharmacodynamic modeling of the electroencephalogram effects of scopolamine in healthy volunteers. J Clin Pharmacol 2001;41(1):51-60.

Fuchs M, Kastner J, Wagner M, Hawes S, Ebersole JS. A standardized boundary element method volume conductor model. Clin Neurophysiol 2002;113:702-12.

Galluzzi S, Marizzoni M, Babiloni C, Albani D, Antelmi L, Bagnoli C, et al. Clinical and biomarker profiling of prodromal Alzheimer's disease in workpackage 5 of the Innovative Medicines Initiative PharmaCog project: a 'European ADNI study'. J Intern Med 2016;279(6):576-91. https://doi.org/10.1111/joim.12482.

Gerrard P, Malcolm R. Mechanisms of modafinil: a review of current research. Neuropsychiatr Dis Treat 2007;3(3):349-64.

Gianotti LR, Künig G, Lehmann D, Faber PL, Pascual-Marqui RD, Kochi K, et al. Correlation between disease severity and brain electric LORETA tomography in Alzheimer's disease. Clin Neurophysiol 2007;118(1):186-96.

Gianotti LR, Künig G, Faber PL, Lehmann D, Pascual-Marqui RD, Kochi K, et al. Rivastigmine effects on EEG spectra and three-dimensional LORETA functional imaging in Alzheimer's disease. Psychopharmacology (Berl) 2008;198 (3):323-32. https://doi.org/10.1007/s00213-008-1111-1.

Giaquinto S, Nolfe G. The EEG in the normal elderly: a contribution to the interpretation of aging and dementia. Electroencephalogr Clin Neurophysiol 1986;63(6):540-6.

Groeger JA, Viola AU, Lo JC, von Schantz M, Archer SN, Dijk DJ. Early morning executive functioning during sleep deprivation is compromised by a PERIOD3 polymorphism. Sleep 2008;31(8):1159-67.

Holschneider DP, Waite JJ, Leuchter AF, Walton NY, Scremin OU. Changes in electrocortical power and coherence in response to the selective cholinergic immunotoxin 192 IgG-saporin. Exp Brain Res 1999;126:270-80.

Horacek J, Brunovsky M, Novak T, Tislerova B, Palenicek T, Bubenikova-Valesova V, et al. Subanesthetic dose of ketamine decreases prefrontal theta cordance in healthy volunteers: implications for antidepressant effect. Psychol Med 2010:40(9):1443-51.

Horváth A, Szücs A, Barcs G, Noebels JL, Kamondi A. Epileptic seizures in alzheimer disease: a review. Alzheimer Dis Assoc Disord. 2016;30(2):186-92. https://doi. org/10.1097/WAD.0000000000000134.

Huang C, Wahlund LO, Dierks T, Julin P, Winblad B, Jelic V. Discrimination of Alzheimer's disease and mild cognitive impairment by equivalent EEG sources: a cross-sectional and logitudinal study. Clin Neurophysiol 2000;11:1961-7.

Hughes SW, Crunelli V. Thalamic mechanisms of EEG alpha rhythms and their pathological implications. Neuroscientist 2005;11(4):357-72.

James LM, Iannone R, Palcza J, Renger JJ, Calder N, Cerchio K, et al. Effect of a novel histamine subtype-3 receptor inverse agonist and modafinil on EEG power spectra during sleep deprivation and recovery sleep in male volunteers. Psychopharmacology (Berl) 2011;215:643-53.

Jelic V, Shigeta M, Julin P, Almkvist O, Winblad B, Wahlund LO. Quantitative electroencephalography power and coherence in Alzheimer's disease and mild cognitive impairment. Dementia 1996;7(6):314-23.

Jeong J. EEG dynamics in patients with Alzheimer's disease. Clin Neurophysiol 2004:115:1490-505.

Johannsson M, Snaedal J, Johannesson GH, Gudmundsson TE, Johnsen K. The acetylcholine index: an electroencephalographic marker of cholinergic activity in the living human brain applied to Alzheimer's disease and other dementias. Dement Geriatr Cogn Disord 2015;39(3-4):132-42. https://doi.org/10.1159/ $\underline{000367889}$.

Jones BE. Activity, modulation and role of basal forebrain cholinergic neurons innervating the cerebral cortex. Prog Brain Res. 2004;145:157-69.

Jovicich J, Babiloni C, Ferrari C, Marizzoni M, Moretti DV, Del Percio C, et al. Twoyear longitudinal monitoring of amnestic mild cognitive impairment patients with prodromal Alzheimer's disease using topographical biomarkers derived 
from functional magnetic resonance imaging and electroencephalographic activity. J Alzheimers Dis 2019;69:15-35.

Jurcak V, Tsuzuki D, Dan I. 10/20, 10/10, and 10/5 systems revisited: their validity as relative head-surface-based positioning systems. Neuroimage 2007:34:1600-11.

Kalmbach A, Hedrick T, Waters J. Selective optogenetic stimulation of cholinergic axons in neocortex. J Neurophysiol 2012;107(7):2008-19. https://doi.org/ 10.1152/jn.00870.2011.

Killgore WD, Kahn-Greene ET, Grugle NL, Killgore DB, Balkin TJ. Sustaining executive functions during sleep deprivation: a comparison of caffeine, dextroamphetamine, and modafinil. Sleep. 2009;32(2):205-16.

Killgore WD, Muckle AE, Grugle NL, Killgore DB, Balkin TJ. Sex differences in cognitive estimation during sleep deprivation: effects of stimulant countermeasures. Int J Neurosci 2008;118(11):1547-57.

Kolev V, Yordanova J, Basar-Eroglu C, Basar E. Age effects on visual EEG responses reveal distinct frontal alpha networks. Clin Neurophysiol 2002;113(6): 901-10.

Lehmann C, Koenig T, Jelic V, Prichep L, John RE, Wahlund LO, et al. Application and comparison of classification algorithms for recognition of Alzheimer's disease in electrical brain activity (EEG). J Neurosci Methods 2007;161(2):342-50.

Leuchter AF, Cook IA, Newton TF, Dunkin J, Walter DO, Rosenberg-Thompson S, et al. Regional differences in brain electrical activity in dementia: use of spectral power and spectral ratio measures. Electroencephalogr Clin Neurophysiol 1993;87(6):385-93.

Liem-Moolenaar M, de Boer P, Timmers M, Schoemaker RC, van Hasselt JG, Schmidt $S$, et al. Pharmacokinetic-pharmacodynamic relationships of central nervous system effects of scopolamine in healthy subjects. Br J Clin Pharmacol 2011;71 (6):886-98. https://doi.org/10.1111/i.1365-2125.2011.03936.x.

Lin CW, Bianchi BR, Miller TR, Stashko MA, Wang SS, Curzon P, et al. Persistent activation of the dopamine D1 receptor contributes to prolonged receptor desensitization: studies with A-77636. J Pharmacol Exp Ther 1996;276 (3):1022-9.

Lo JC, Groeger JA, Santhi N, Arbon EL, Lazar AS, Hasan S, et al. Effects of partial and acute total sleep deprivation on performance across cognitive domains, individuals and circadian phase. PLoS One. 2012;7(9). https://doi.org/10.1371/ journal.pone.0045987 e45987.

Lopes da Silva FL. EEG and MEG: relevance to neuroscience. Neuron 2013;80 (5):1112-28.

Lörincz ML, Crunelli V, Hughes SW. Cellular dynamics of cholinergically induced alpha $(8-13 \mathrm{~Hz})$ rhythms in sensory thalamic nuclei in vitro. J Neurosci 2008;28 (3):660-71.

Lörincz ML, Kékesi KA, Juhász G, Crunelli V, Hughes SW. Temporal framing of thalamic relay-mode firing by phasic inhibition during the alpha rhythm. Neuron. 2009;63(5):683-96.

Mander BA, Reid KJ, Baron KG, Tjoa T, Parrish TB, Paller KA, et al. EEG measures index neural and cognitive recovery from sleep deprivation. J Neurosci 2010;30 (7):2686-93. https://doi.org/10.1523/INEUROSCI.4010-09.2010.

Mazziotta JC, Toga AW, Evans A, Fox P, Lancaster J. A probabilistic atlas of the human brain: theory and rationale for its development. The International Consortium for Brain Mapping (ICBM). Neuroimage 1995;2:89-101.
Moretti DV, Babiloni C, Binetti G, Cassetta E, Dal Forno G, Ferreric F, et al. Individual analysis of EEG frequency and band power in mild Alzheimer's disease. Clin Neurophysiol. 2004;115:299-308.

Moretti DV, Babiloni F, Carducci F, Cincotti F, Remondini E, Rossini PM, et al Computerized processing of EEG-EOG-EMG artifacts for multi-centric studies in EEG oscillations and event-related potentials. Int J Psychophysiol 2003;47 (3):199-216.

Neufeld MY, Rabey MJ, Parmet Y, Sifris P, Treves TA, Korczyn AD. Effects of a single intravenous dose of scopolamine on the quantitative EEG in Alzheimer's disease patients and age-matched controls. Electroencephalogr Clin Neurophysiol. 1994;91(6):407-12.

Nobili F, Copello F, Vitali P, Prastaro T, Carozzo S, Perego G, et al. Timing of disease progression by quantitative EEG in Alzheimer's patients. J Clin Neurophysiol. 1999;16(6):566-73.

Nunez PL. Removal of reference electrode and volume conduction effects by spatial deconvolution of evoked potentials using a three-concentric sphere model of the head. Electroencephalogr Clin Neurophysiol Suppl 1987;39:143-8.

Ommundsen N, Engedal K, Øksengård AR. Validity of the quantitative EEG statistical pattern recognition method in diagnosing Alzheimer'sdisease. Dement Geriatr Cogn Disord 2011;31(3):195-201. https://doi.org/10.1159/000324878.

Pascual-Marqui RD, Michel CM, Lehmann D. Low resolution electromagnetic tomography: a new method for localizing electrical activity in the brain. Int Psychophysiol 1994:18:49-65.

Pascual-Marqui RD. Discrete 3D distributed, linear imaging methods of electric neuronal activity. Part 1: exact, zero error localization. Math Phys 2007:1-16.

Rodriguez G, Copello F, Nobili F, Vitali P, Perego G, Nobili F. EEG spectral profile to stage Alzheimer's disease. Clin Neurophysiol 1999;110:1831-7.

Saletu M, Anderer P, Saletu-Zyhlarz GM, Mandl M, Arnold O, Zeitlhofer J, et al. EEGtomographic studies with LORETA on vigilance differences between narcolepsy patients and controls and subsequent double-blind, placebo-controlled studies with modafinil. J Neurol 2004;251(11):1354-63.

Saletu M, Anderer P, Semlitsch HV, Saletu-Zyhlarz GM, Mandl M, Zeitlhofer J, et al. Low-resolution brain electromagnetic tomography (LORETA) identifies brain regions linked to psychometric performance under modafinil in narcolepsy. Psychiatry Res 2007;154(1):69-84.

Scarmeas N, Honig LS, Choi H, Cantero J, Brandt J, Blacker D, et al. Seizures in Alzheimer disease: who, when, and how common? Arch Neurol 2009;66 (8):992-7. https://doi.org/10.1001/archneurol.2009.130.

Snaedal J, Johannesson GH, Gudmundsson TE, Gudmundsson S, Pajdak TH, Johnsen K. The use of EEG in Alzheimer's disease, with and without scopolamine - a pilot study. Clin Neurophysiol 2010;121(6):836-41.

Szava S, Valdes P, Biscay R, Galan L, Bosch J, Clark I, et al. High resolution quantitative EEG analysis. Brain Topogr 1994;6(3):211-9.

Van Dongen HP, Dinges DF. Sleep, circadian rhythms, and psychomotor vigilance. Clin Sports Med 2005;24(2):237-49.

Vossel KA, Beagle AJ, Rabinovici GD, Shu H, Lee SE, Naasan G, et al. Seizures and epileptiform activity in the early stages of Alzheimer disease. JAMA Neurol 2013;70(9):1158-66. https://doi.org/10.1001/jamaneurol.2013.136.

Widlöcher D. Les Nouvelles Cartes de la psychanalyse. 1996. Edition: Paris: Odile Jacob, 1996 (27-Mesnil-sur-l'Estrée: Impr. Firmin-Didot). 